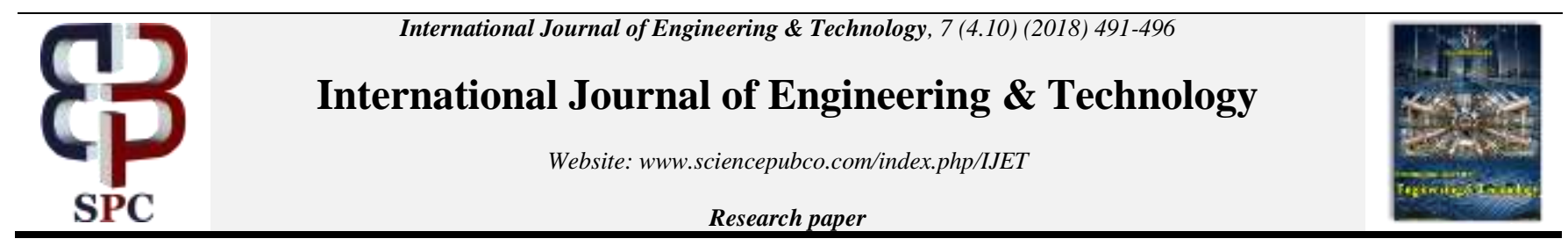

\title{
Flow of a Herschel-Bulkley Fluid in a Channel with Elastic Walls
}

\author{
S.Sreenadh ${ }^{1}$, B.Sumalatha ${ }^{1}$ and A.N.S.Srinivas ${ }^{2^{*}}$ \\ ${ }^{1}$ Department of Mathematics, Sri Venkateswara University, Tirupati, 517 502, A.P., India. \\ ${ }^{2}$ Department of Mathematics, School of Advanced Sciences, VIT, Vellore, 632 014, T.N., India. \\ *Corresponding authorE-mail: anssrinivas@vit.ac.in, akkirajusrinivas@gmail.com
}

\begin{abstract}
In order to model the blood flow through an artery in presence of catheter, we considered a steady, laminar, incompressible, Poiseuille flow of a Herschel-Bulkley fluid between two horizontal parallel elastic walls. The power law index $(n)$ and yield stress $\left(\tau_{0}\right)$ are the two parameters of the Herschel - Bulkley fluid. By giving different values for the above mentioned parameters, we get the Newtonian, Bingham and Power-law fluids as special cases. The exact solutions for the flow quantities such as velocity, plug flow velocity and flux are derived. The flux is determined as a function of inlet, outlet, external pressures and the elastic property of the channel. The effect of elastic parameters on flux variation is analyzed. Further when $n=1$ and $\tau_{0}=0$ our results qualitatively agree with those of Rubinow and Keller [2]. In addition, velocity of the Herschel- Bulkley fluid flow is expressed in terms of elastic parameters.
\end{abstract}

Keywords: Poiseuille flow, Herschel-Bulkley fluid, elastic parameters

\section{Introduction}

In recent days, the flow of Newtonian and non-Newtonian fluids through channels/tubes contributes significant attention due to their wide applications in engineering and medical sciences. The flow geometry plays an important role in understanding the characteristics of different fluid flows. Most of the earlier studies are concentrated on rigid channels and tubes. In biological systems, the flow geometries with elastic nature is more adequate when compared to the rigid boundaries. Since, most of the physiological systems are elastic in nature. So the non-Newtonian fluid flow through elastic walls gives some important applications like blood flow in a small blood vessel, lymphatic vessel and cardiovascular systems to understand the evolution of pathogen due to vessel deformation. To understand the rheological properties of physiological fluids in living organisms, the elastic properties of flow geometries are taken in to the consideration. The experimental studies reveal that velocity in blood vessels largely depends on the elastic nature of the wall.

Roach and Burton [1] conducted an experiment on human external iliac artery to study the static pressure-volume relation as tension versus length curve. Rubinow and Keller [2] explained the detailed analysis applications blood flow by considering viscous fluid flow through elastic tubes. The non-Newtonian behaviour of blood at lower shear rates was analysed by Pedley and Fung [3]. Newtonian fluid flow in an elastic tube was discussed by Fung [4]. Mazumdar [5] presented the analysis of non-Newtonian fluid flow through elastic tube by expressing the tension relation as an exponential curve. Taha and Sochi [6] discussed the lubrication approximation to interpret the flow behaviour of Newtonian fluid and Power-Law fluids in elastic tubes. Scott Blair et al. [7] reported that Herschel-Bulkley fluid behaves like cow's blood. Nonlinear analysis for oscillatory flow through elastic tube and its applications are derived by Sankar and Jayraman [8]. The unsteady flow of a viscous, incompressible fluid in a tube with an elastic insertion is studied by Pedrizzetti [9]. Vajravelu et al. [10] investigated the peristaltic transport of a Herschel- Bulkley fluid in a channel. Mathematical model is proposed by Vajravelu et al. [11] to understand the Herschel- Bulkley fluid flow in an elastic tube. Ali et al. [12] presented a numerical study creeping flow of Herschel-Bulkley fluids in collapsible channels. Creeping flow of a Herschel-Bulkley fluid with pressure-dependent material moduli is discussed by Fusi and Rosso [13].

In this paper we consider the flow of Herschel - Bulkley fluid in a plane analogue with elastic walls. The flux of the channel is obtained as a function of elastic parameters. The influence of various pertinent parameters on variation of flux along the walls of the channel for a Herschel - Bulkley fluid in an elastic walls are calculated and interpreted through graphs.

\section{Mathematical Formulation:}

The governing equations for the flow are

$$
\operatorname{div} V=0
$$

$\operatorname{div} \sigma+\rho f=\rho \frac{d V}{d t}$

Where $V$ is the velocity, $f$ is the body force per unit mass, $\rho$ is the density, $\frac{d}{d t}$ the material derivative and $\sigma$ is the Cauchy stress defined by 


$$
\begin{aligned}
& \sigma=-p I+T \\
& T=2 \mu D+S \\
& S=2 \eta D
\end{aligned}
$$

$D$ is the symmetric part of the velocity gradient, which is defined by $D=\frac{1}{2}\left[L+L^{T}\right]$ and $L=\operatorname{grad} V$ also, $-p I$ denotes the intermediate part of the stress due to the constraint of incompressibility. $\mu$ and $\eta$ are the viscosities.

\section{Flow Geometry and Governing Equations:}

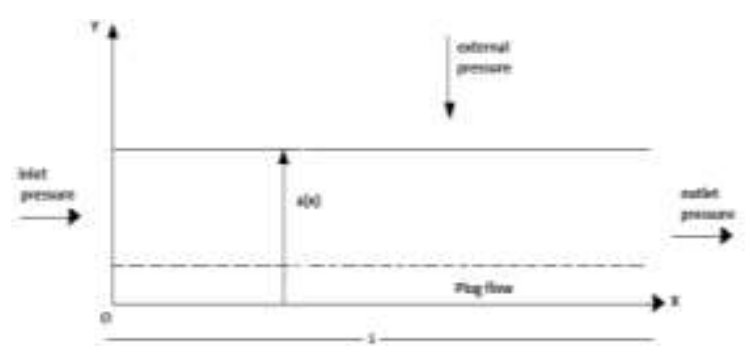

Fig. 1: Physical Model

Consider the steady, laminar, incompressible, Poiseuille flow of a Herschel - Bulkley fluid in a horizontal channel with elastic plane analogue. $L$ and $a(x)$ be the length and half width of the channel respectively. $\mu$ is the viscosity of the Herschel- Bulkley fluid and $\tau_{y x}$ is the shear stress. The region between $y=0$ and $y=y_{0}$ is called a plug flow region, $\left|\tau_{x y}\right| \leq \tau_{0}$. In the region between $y=y_{0}$ and $y=a(x)$, we have $\left|\tau_{x y}\right| \geq \tau_{0}$. The momentum equation governing the flow is

$\frac{\partial}{\partial y}\left(\tau_{y x}\right)=\frac{\partial p}{\partial x}$

where $\tau_{y x}=\mu\left(-\frac{\partial u}{\partial y}\right)^{n}+\tau_{0}$

here $u$ is the axial velocity and $p$ is the pressure. The corresponding boundary conditions are

at $y=a(x): u=\frac{\partial \psi}{\partial y}=0$

at $y=0: u_{y}=\frac{\partial^{2} \psi}{\partial y^{2}}=0$

at $y=a(x): \psi=q$

at $y=0: \psi=0$

at $y=0: \tau_{y x}=0$

where $\psi$ is the stream function and $\tau_{0}$ is the yield stress.
To solve equations (6) and (7) subject to the boundary conditions in (8), we use the following non-dimensional quantities:

$\bar{u}=\frac{u}{U} ; \bar{x}=\frac{x}{L} ; \bar{y}=\frac{y}{a_{0}} ; \bar{a}=\frac{a(x)}{a_{0}}, \bar{p}=\frac{p y_{0}{ }^{n+1}}{L \mu U^{n}} ;$

$\bar{\tau}_{0}=\frac{\tau_{0}}{\mu\left(U / a_{0}\right)^{n}} ; \bar{\tau}_{y x}=\frac{\tau_{y x}}{\mu\left(U / a_{0}\right)^{n}} ; \bar{\psi}=\frac{\psi}{a_{0} U} ; \bar{q}=\frac{q}{a_{0} U}$

Here $U$ is the average velocity, $a_{0}$ is the width of the channel in the absence of elasticity and $n$ is the power law index.

By using the non-dimensional quantities given in equation (9), the momentum equation become (after removing the bars)

$\frac{\partial}{\partial y}\left(\tau_{y x}\right)=-P$

where $P=-\frac{\partial p}{\partial x}$ and $\tau_{y x}=\left(-\frac{\partial u}{\partial y}\right)^{n}+\tau_{0}$

The dimensionless boundary conditions are as follows:

at $y=a(x): u=\frac{\partial \psi}{\partial y}=0$

at $y=0: u_{y}=\frac{\partial^{2} \psi}{\partial y^{2}}=0$

at $y=a(x): \psi=q$

at $y=0: \psi=0$

at $y=0: \tau_{y x}=0$

\section{Solution of the Problem:}

Solving equation (10) with the boundary conditions (12), we obtain the velocity as

$u=\frac{1}{\left(\frac{1}{n}+1\right) P^{[}}\left[\left(P a-\tau_{0}\right)^{\frac{1}{n}+1}-\left(P y-\tau_{0}\right)^{\frac{1}{n}+1}\right]$

By using the boundary condition $\psi_{y y}=0$ at $y=y_{0}$, the upper limit of the plug flow region is derived as

$y_{0}=\frac{\tau_{0}}{P}$

Using the condition $\tau_{y x}=\tau_{h}$ at $y=a(x)$, we get

$P=\frac{\tau_{h}}{a}$

Hence $\frac{y_{0}}{a}=\frac{\tau_{0}}{\tau_{h}}=\tau \quad$ where $0<\tau<1$ 
By taking $y=y_{0}$ in equation (13), we get velocity for the plug flow regions as

$$
u_{p}=\frac{1}{\left(\frac{1}{n}+1\right) P}\left(P a-\tau_{0}\right)^{\frac{1}{n}+1}
$$

The flux $q$ is given by

$$
q=\int_{0}^{y_{0}} u_{p} d y+\int_{y_{0}}^{a} u d y=\frac{(1-\tau)^{\frac{1}{n}+1}\left(1+\frac{1}{n}+\tau\right)}{\left(\frac{1}{n}+1\right)\left(\frac{1}{n}+2\right)} a^{\frac{1}{n}+2} P^{\frac{1}{n}}
$$

Due to the pressure difference between the outside of the walls and fluid, there is a corresponding change in width of the channel $a(x)$. This shows that the flow follows the famous Poiseuille law which states the flux is expressed as a linear function of pressure difference between inlet and outlet of the channel. Thus, we have

$$
q=\sigma_{1}\left(p-p_{0}\right) P^{\frac{1}{n}}
$$

From equations (18) and (19), we get the conductivity of the channel as

$$
\sigma_{1}\left(p-p_{0}\right)=G a^{\frac{1}{n}+2}
$$

where $G=\frac{(1-\tau)^{\frac{1}{n}+1}\left(1+\frac{1}{n}+\tau\right)}{\left(\frac{1}{n}+1\right)\left(\frac{1}{n}+2\right)}$

Integrating the equation (19) w.r.t. $x$ from $x=0$ and applying inlet condition $p(0)=p_{1}$, we get

$$
q^{n} x=-\int_{\mathrm{p}(0)-p_{0}}^{\mathrm{p}(x)-p_{0}}\left(\sigma_{1}\left(p^{\prime}\right)\right)^{n} d p^{\prime}
$$

where $p^{\prime}=p(x)-p_{0}$

To find flux $Q$, we set $x=1$ and $p(1)=p_{2}$, hence we get

$$
\begin{aligned}
& q^{n}=\int_{\mathrm{p}_{2}-p_{0}}^{\mathrm{p}_{1}-p_{0}}\left(\sigma_{1}\left(p-p_{0}\right)\right)^{n} d p^{\prime} \\
& q^{n}=G^{n} \int_{\mathrm{p}_{2}-p_{0}}^{\mathrm{p}_{1}-p_{0}} a^{2 n+1} d p^{\prime}
\end{aligned}
$$

If the stress or tension $T(a)$ in the walls of the channel is known as a function of $a$, then $a\left(p^{\prime}\right)$ can be found by the equilibrium condition,

$$
\frac{T(a)}{a}=p-p_{0}
$$

Now, it is necessary to know how the radius of a tube varies with pressure. Roach \& Burton [1] determined the static pressurevolume relation of a $4 \mathrm{~cm}$ long piece of the human external iliac artery, and converted it into a tension versus length curve. Using least squares method Rubinow and Keller [2] gave the following equation:

$$
T(a)=t_{1}(a-1)+t_{2}(a-1)^{5}
$$

where $t_{1}=13$ and $t_{2}=300$

From equations (25) and (26), we get

$d p^{\prime}=\left[\frac{t_{1}}{a_{2}}+t_{2}\left(4 a^{3}-15 a^{2}+20 a-10+\frac{1}{a^{2}}\right)\right] d a$

By substituting the equation (27) in equation (24) and then solving the integral, we get the flux of the fluid as

$q^{n}=G^{n} \int_{\mathrm{p}_{2}-p_{0}}^{\mathrm{p}_{1}-p_{0}}\left[\begin{array}{l}t_{1}\left(a^{2 n-1}\right)+t_{2}\left(4 a^{2 n+4}-15 a^{2 n+3}\right. \\ \left.+20 a^{2 n+2}-10 a^{2 n+1}+a^{2 n-1}\right)\end{array}\right] d a$

$q=G\left[g\left(a_{1}\right)-g\left(a_{2}\right)\right]^{\frac{1}{n}}$

$g(a)=t_{1} \frac{a^{2 n}}{2 n}+t_{2}\left(4 \frac{a^{2 n+5}}{2 n+5}-15 \frac{a^{2 n+4}}{2 n+4}+20 \frac{a^{2 n+3}}{2 n+3}-10 \frac{a^{2 n+2}}{2 n+2}+\frac{a^{2 n}}{2 n}\right)$

where $a_{1}=a\left(\mathrm{p}_{1}-p_{0}\right), a_{2}=a\left(\mathrm{p}_{2}-p_{0}\right)$

From equation (10), we get

$\frac{\partial^{4} \psi}{\partial y^{4}}=0$

Integrating equation (31) by using the boundary conditions given in equation (12), we get the stream function as

$\psi=\frac{q\left(\frac{1}{n}+2\right)}{a^{\frac{1}{n}+2}\left(\frac{1}{n}+1\right)}\left[a^{\frac{1}{n}+1} y-\frac{y^{\frac{1}{n}+2}}{m+2}\right]$

Hence the velocity field in terms of elastic parameter $t_{1}$ and $t_{2}$ is

$u=\frac{q\left(\frac{1}{n}+2\right)}{a^{\frac{1}{n}+2}\left(\frac{1}{n}+1\right)}\left[a^{\frac{1}{n}+1}-y^{\frac{1}{n}+1}\right]$

\section{Results and Discussions:}

In the present paper, the flow of Herschel-Bulkley fluid in a channel with elastic walls is studied. The analytic expressions for axial velocity and stream function are derived. The relation between volume flow rates is obtained. The influence of various pertinent parameters on flow quantities are analysed through graphs.

The effect of physical parameters such as power-law index $n$, yield stress $\tau$, elastic parameters $t_{1}$ and $t_{2}$ on variation of flux is represented in Figures 2 to 7 respectively. To calculate flux, we used the fixed values for different parameters 
$t_{1}=13, t_{2}=300, n=1.2, \tau=0.2$ except for varied values as shown in figures. Figure 2 shows the variation in flux along the channel width for different types of biofluids. It is noticed that the flux is more for Newtonian case when compared to nonNewtonian case. Figure 3 denotes the influence of yield stress on flux. It is clear that increasing yield stress reduces the flux.

Figure 4 and 5 illustrates the variation of flow rate for different values of elastic parameters $t_{1}$ and $t_{2}$ respectively. It is found that the flux enhances with growing values of elastic parameters. Figure 6 represents that the flux decreases with increasing $n$ for shear thinning case where the opposite behavior is observed in the case of shear thickening which is shown in Figure 7. Figures 8 and 9 denotes change in flux for different values of $p_{2}-p_{0}$ and $p_{1}-p_{0}$ respectively. It is found that the flux reduces for increasing outlet and external pressure difference where it increases for increasing inlet and external pressure difference.

The effect of physical parameters such as power-law index $n$, yield stress $\tau$, elastic parameters $t_{1}$ and $t_{2}$ on axial velocity is represented in Figures 10 to 13 respectively. To calculate velocity we used the fixed values for different parameters $t_{1}=13, t_{2}=300, n=0.8, \tau=0.5$ except for varied values as shown in figures. The variation in velocity for different values of $t_{1}$ and $t_{2}$ are presented in Figures 10 and 11 respectively. It is clear that increasing elastic parameters decreases the velocity of the fluid. The effect of fluid behavior index $n$ on fluid velocity is illustrated in Figure 12. That is the fluid velocity gets reduced with increasing $n$. The effect of yield stress on velocity is shown in Figure 13. It is observed that fluid velocity decreases with increasing yield stress.

\section{Conclusions:}

In the present paper, the flow of Herschel-Bulkley fluid in a channel with elastic walls is investigated. The solutions for axial velocity, stream function and flux are obtained analytically. The influence of different parameters on flow quantities are analyzed graphically.

i) The flux of Newtonian fluids in a channel with elastic walls is more when compared to non- Newtonian fluids.

ii) The flux of Herschel-Bulkley fluid decreases with increasing yield stress where it is decreases with higher values of elastic parameters.

iii) The effect of power-law index on flux for both shear thinning and shear thickening fluids are analyzed.

iv) The flux decreases with increasing outlet and external pressure difference where the opposite behavior is observed in the case of inlet and external pressure difference.

v) The fluid velocity increases with increasing elastic parameter and decreases for increasing power-law index and yield stress.

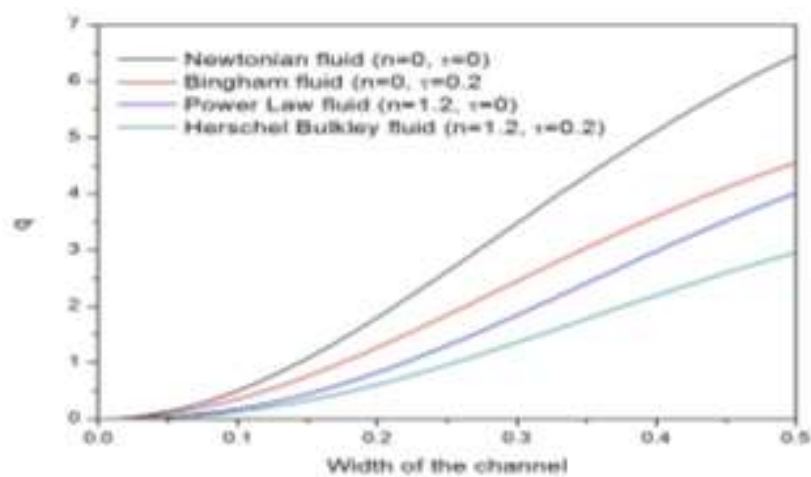

Fig. 2: The flux vs channel width for different fluids.

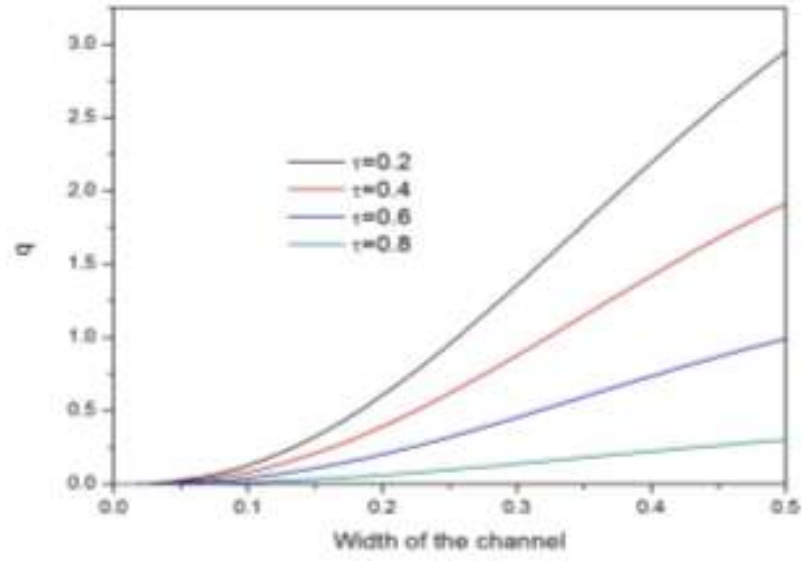

Fig. 3: The flux vs channel width for different values of yield stress.

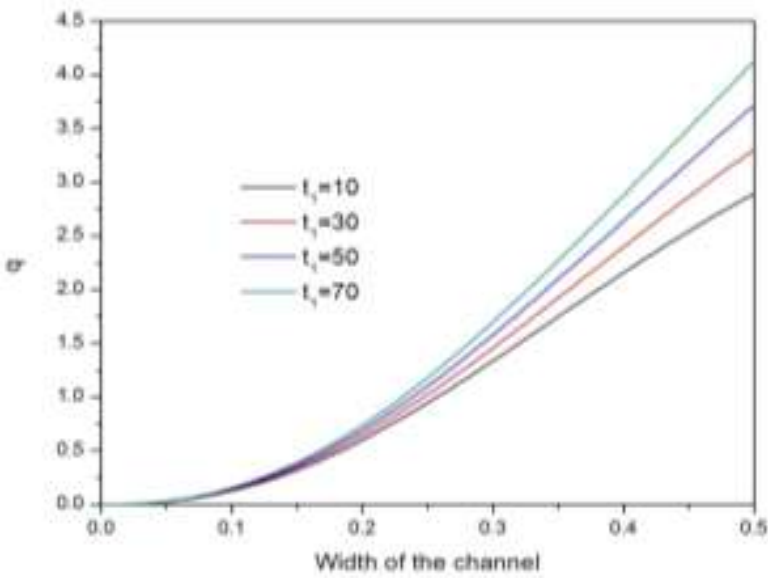

Fig. 4: The flux vs. channel width for different values of $t_{1}$

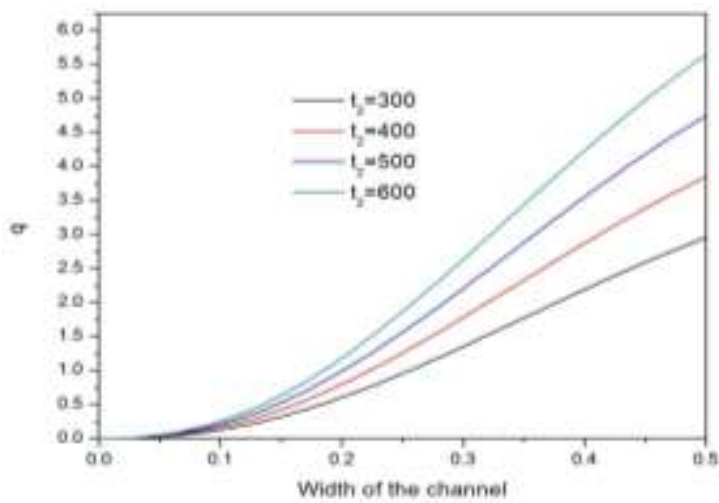

Fig. 5: The flux vs. channel width for different values of $t_{2}$

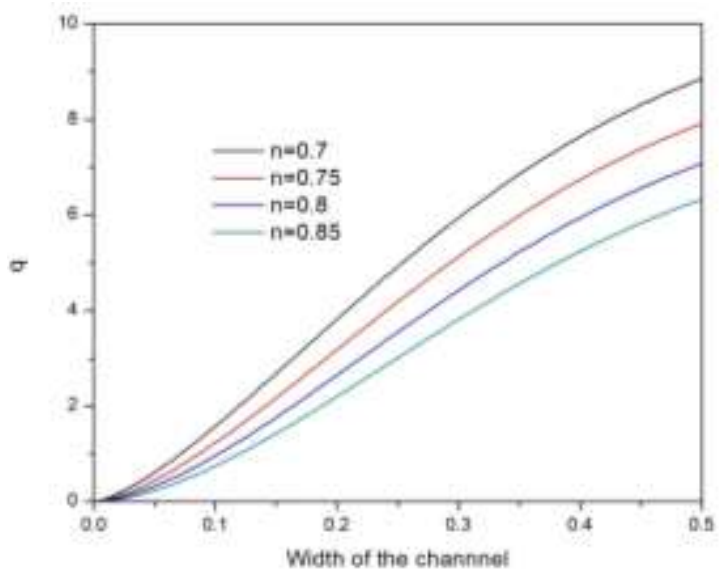

Fig. 6: The flux vs. channel width for different values of $n$ (shear thinning fluid) 


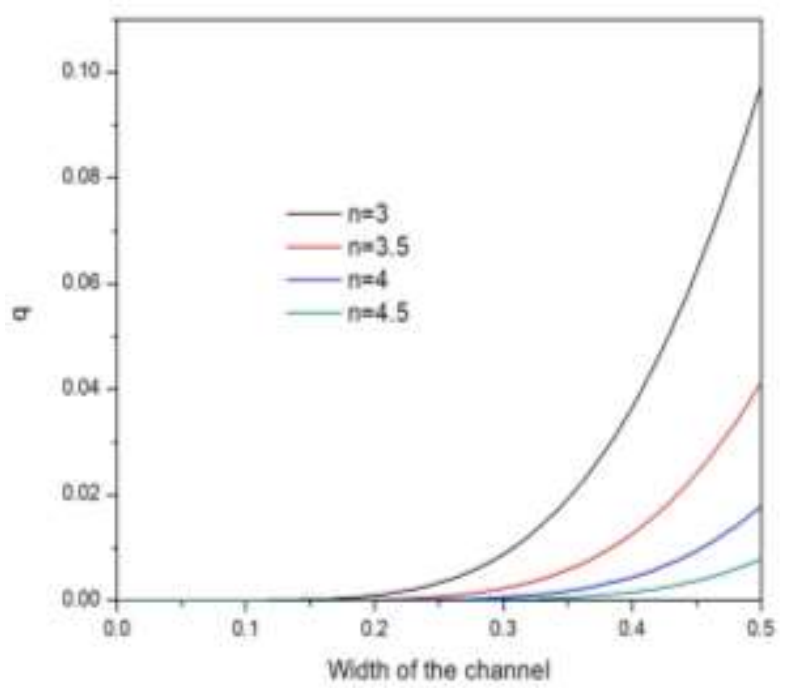

Fig.7: The flux vs channel width for different values of $n$ (shear thickening)

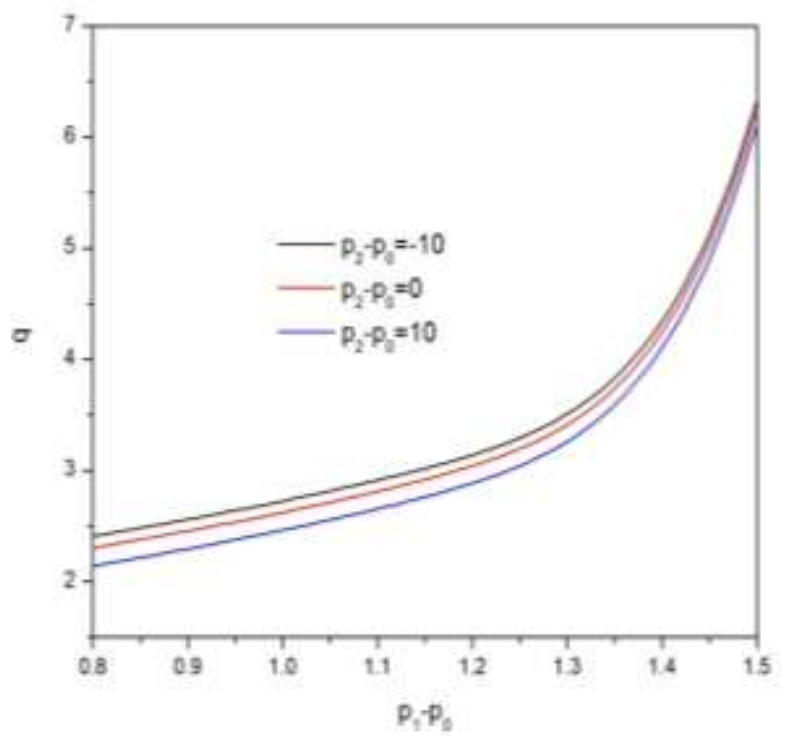

Fig. 8: The flux vs. inlet pressure for different values of outlet pressure

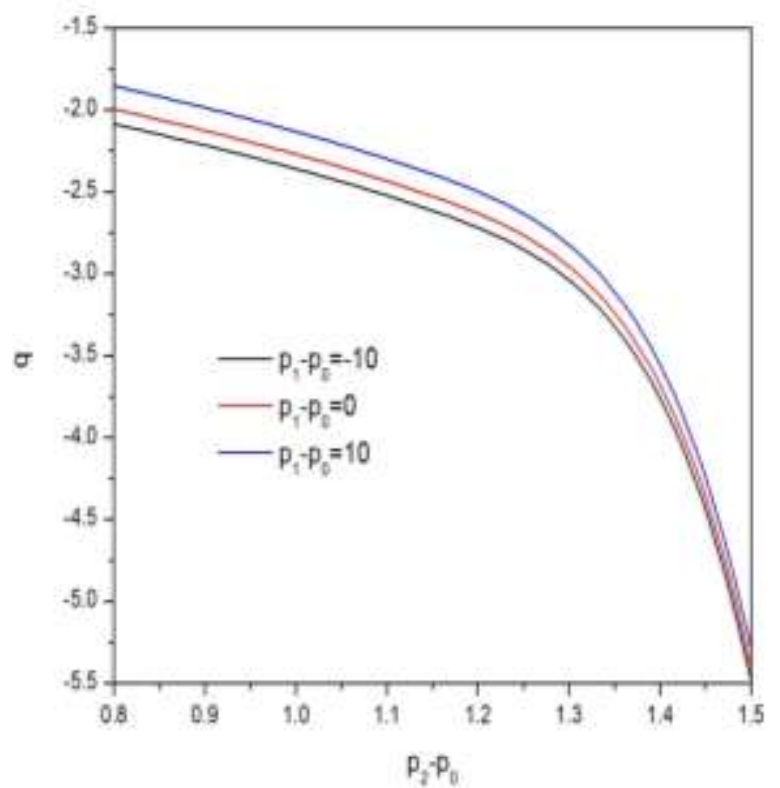

Fig. 9: The flux vs. outlet pressure for different values of inlet pressure

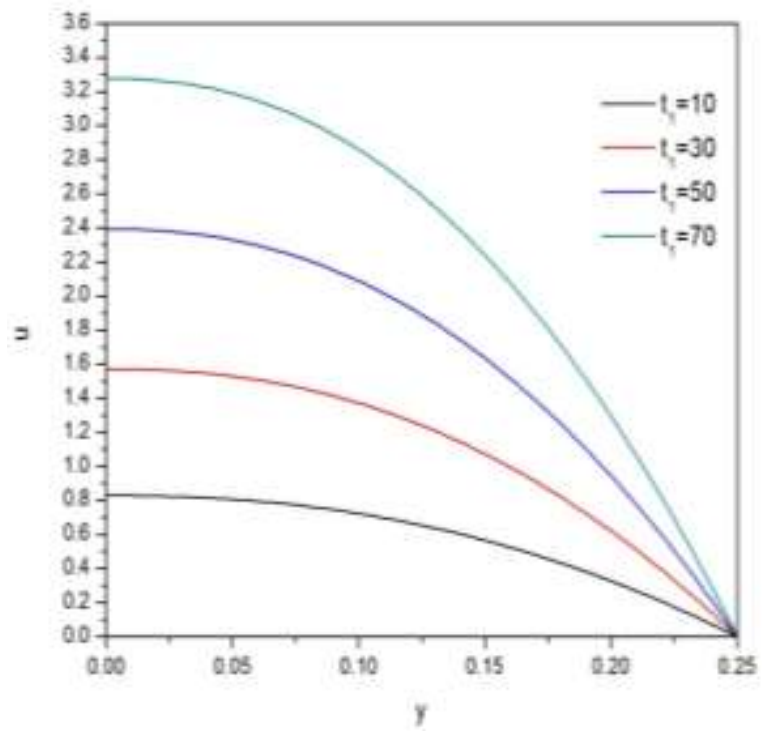

Fig. 10: The velocity vs $y$ for different values of $t_{1}$

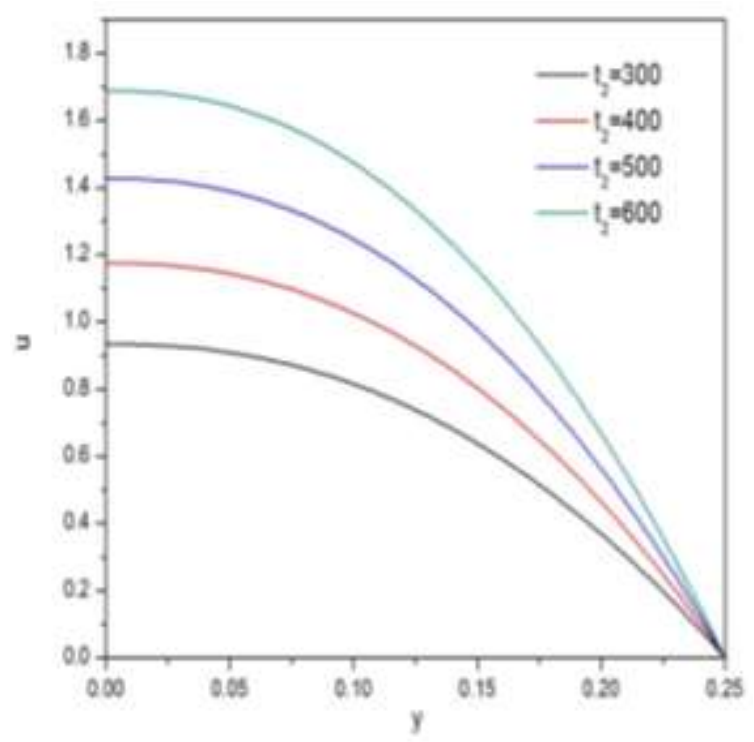

Fig. 11: The velocity vs. $y$ for different values of $t_{2}$

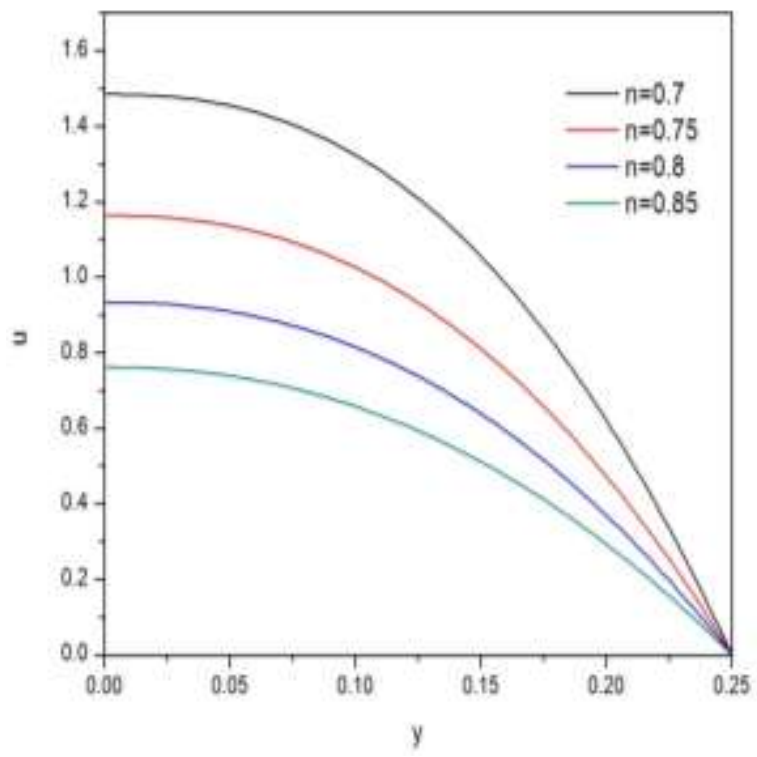

Fig. 12: The velocity vs. $y$ for different values of $n$ 


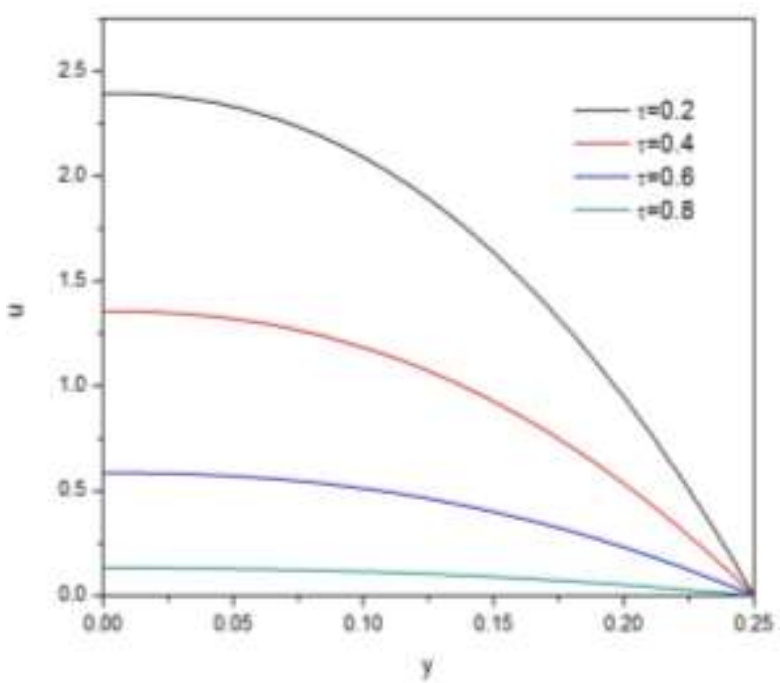

Fig. 13: The velocity vs. $y$ for different values of $\tau$

\section{References}

[1] Roach, M. R., \& Burton, A. C. "The reason for distensibility curves of arteries". Canadian Journal of Biochemistry and Physiology, Vol. 35(8) (1957) pp. 681-690. http://doi: 10.1139/o57-080.

[2] Rubinow, S. I., \& Keller, J. B.” Flow of a viscous fluid through an elastic tube with applications to blood flow". Journal of Theoretical Biology, Vol. 35(2) (1972), pp. 299-313. https://doi.org/10.1016/0022-5193(72)90041-0.

[3] Pedley, T. J., \& Fung Y .C.'The fluid mechanics of large blood vessels". Cambridge University press (1980).

[4] Fung, Y. C., "Biodynamics", Springer-Verlag, Newyork, Berlin Hildelberd, Tokyo (1984).

[5] Mazumdar, N. J., "Biofluid Mechanics". World Scientific publishing, Singapore (1992).

[6] Taha Sochi, "The flow of Newtonian and Power-law fluids in elastic tubes", Intern. Journal of Non-linear Mechanics, Vol.67 (2014), pp. 245 - 250. https://doi.org/10.1016/j.ijnonlinmec.2014.09.013.

[7] Scott Blair G. W. S. "An equation for the flow of blood, Plasma and serum through glass Capillaries”. Nature, Vol. 183 (1959), pp. 613-614. http://dx.doi.org/10.1038/183613a0

[8] Shankar, A \& Jayaraman, G.,"Non-linear analysis of oscillatory flow in the annulus of an elastic tube: Applications to catheterized artery". Physics of Fluids, Vol. 13(10) (2001), pp. 2901-2911. https://doi.org/10.1063/1.1389285.

[9] Pedrizzetti, G., "Fluid flow in a tube with elastic membrane insertion", Journal of Fluid Mechanics, Vol. 375, (1998) pp. 39-64. https://doi.org/10.1017/S0022112098002316

[10] Vajravelu, K., Sreenadh, S., and Ramesh Babu, V., "Peristaltic pumping of a Herschel-Bulkley fluid in a channel". Applied Mathematics and computation, Vol. 169 (1) (2005), pp. 726-735 http://doi.10.1016/j.amc.2004.09.063.

[11] Vajravelu, K., Sreenadh, S., Devaki, P., and Prasad, K. V., (2014) "Mathematical model for aHerschel- Bulkley fluid flow in elastic tube". Central European Journal of Physics, 9(5), 1357-1365. https://doi.org/10.2478/s11534-011-0034-3

[12] Ali Amini, Amir Saman Eghtesad and Kayvan Sadeghy, "Creeping flow of Herschel-Bulkley fluids in collapsible channels: A numerical study". Korea-Australia Rheology Journal, Vol. 28(4) (2016), pp. 255-265. https://doi.org/10.1007/s13367-016-0027-2

[13] Fusi, L., \& Rosso, F., "Creeping flow of a Herschel-Bulkley fluid with pressure-dependent material moduli". European Journal of Applied Mathematics, Vol. 29(2) (2017), pp. 352-368. http://doi $10.1017 /$ S0956792517000183 